\title{
HRJ
}

V.3 n.14 (2022)

Recebido: 20/12/2021

Aceito: 04/01/2022

\section{Abreviação do jejum pré-operatório em cirurgias eletivas: revisão integrativa}

\section{Marcelo Vieira Pio ${ }^{1}$ \\ Lucivane Julia de Queiroz ${ }^{2}$ Marcelo Moreira Corgozinho ${ }^{3}$}

\footnotetext{
${ }^{1}$ Enfermeiro do Programa de Residência Uniprofissional de Enfermagem em Centro Cirúrgico da ESCS/Fepecs. E-mail: piomarcelo536@gmail.com

${ }^{2}$ Preceptora do Programa de Residência Uniprofissional de Enfermagem em Centro Cirúrgico da ESCS/Fepecs. Especialista em Enfermagem de Centro Cirúrgico e CME pelo Instituto Brasileiro de Extensão Educacional. Email: lucivane_julia@ hotmail.com

${ }^{3}$ Tutor e Preceptor do Programa de Residência Uniprofissional de Enfermagem em Centro Cirúrgico da ESCS/Fepecs. Doutor pela Faculdade de Saúde da Universidade de Brasília. E-mail: mmcorgozinho@gmail.com
}

\section{RESUMO}

Objetivo: Descrever, a partir da literatura, as recomendações atuais para a abreviação do jejum pré-operatório em cirurgias eletivas. Método: trata-se de uma revisão integrativa que seguiu as etapas adaptadas do Preferred Reporting Items for Systematic Reviews and MetaAnalyses. As bases de dados utilizadas foram a Literatura Latino-americana e do Caribe em Ciências da Saúde e Medical Literature Analysis and Retrieval System Online, com os Descritores em Ciências da Saúde: “jejum" and "Cuidados pré-operatório" and "procedimentos cirúrgicos eletivos" - entre 2012 a 2021. Resultados: as informações subsidiaram a eleição de três categorias temáticas, a saber: prejuízos do jejum pré-operatório prolongado em cirurgias eletivas, segurança da abreviação do jejum e seus benefícios, e recomendações para a abreviação do jejum pré-operatório. Conclusão: conclui-se que o jejum pré-operatório prologado causa prejuízos os pacientes, enquanto que a abreviação do jejum pré-operatório gera benefício - é seguro a administração de líquidos claros até uma hora antes do procedimento, e de até duas horas com a utilização de solução de carboidrato.

Palavras-chave: Jejum, Cuidados Pré-Operatório, Procedimentos Cirúrgicos Eletivos.

\section{Abbreviation of preoperative fasting in elective surgeries: integrative review}

\begin{abstract}
Objective: To describe, based on the literature, the current recommendations for the abbreviation of preoperative fasting in elective surgeries. Method: this is an integrative review that followed the steps adapted from the Preferred Reporting Items for Systematic Reviews and Meta-Analyses. The databases used were the Latin American and Caribbean Literature on Health Sciences and the Medical Literature Analysis and Retrieval System Online, with the Health Sciences Descriptors: "fasting" and "Preoperative care" and "elective surgical procedures" - between 2012 and 2021. Results: the information supported the election of three thematic categories, namely: damages of prolonged preoperative fasting in elective surgeries, safety of fasting abbreviation and its benefits, and recommendations for
\end{abstract}


preoperative fasting abbreviation -operative. Conclusion: it is concluded that prolonged preoperative fasting causes harm to patients, while the abbreviation of preoperative fasting generates benefits - it is safe to administer clear liquids up to one hour before the procedure, and up to two hours with the use of carbohydrate solution.

Keywords: Fasting, Preoperative Care, Elective Surgical Procedures.

\section{INTRODUÇÃO}

O centro cirúrgico é uma unidade hospitalar onde são realizados procedimentos anestésico-cirúrgicos, diagnósticos e terapêuticos - procedimentos eletivos ou emergenciais que demandam recursos materiais com alta precisão e eficácia ${ }^{1}$. Trata-se de um ambiente de alto risco, onde os processos de trabalho são formados por práticas complexas e interdisciplinares, com grande dependência de uma equipe que trabalha sob muita pressão e estresse. $^{2}$

Em relação aos procedimentos cirúrgicos, é importante destacar que existem inúmeros preparativos tanto para a equipe cirúrgica quanto para o paciente. Para as cirurgias eletivas, por exemplo, é necessária a realização de exames, suspensão de medicamentos em alguns casos, e o jejum pré-operatório no dia da cirurgia. ${ }^{1}$

O uso do jejum em intervenções cirúrgicas tem seus primeiros registros em 1883, se consolidando no século XX - precisamente em 1946 - com o médico obstetra americano Mendelson. O médico analisou casos de óbitos em gestantes relacionados à aspiração de substância gástrica sólida após a indução anestésica geral (Síndrome de Mendelson). Após esse estudo foi estabelecido o jejum pré-operatório com a finalidade de impedir o surgimento de complicações respiratórias resultantes da aspiração de conteúdo gástrico. ${ }^{3,4}$ A prática do jejum pré-operatório seguiu sem modificações até os anos 80, onde começou a sua reestruturação. Com a evolução da medicina e com novas evidências científicas surgiu a necessidade de estudar os procedimentos médicos provenientes de antigas referências. ${ }^{5}$ 
O jejum prolongado é considerado negativo, pois causa alguns riscos e desconfortos ao paciente ${ }^{6}$, como ansiedade; agitação pré-operatória; modificações metabólicas - como a resistência à insulina que resulta em hipoglicemia e na redução da insulina nos tecidos periféricos -, o que bloqueia a captação da glicose que influenciará na recuperação com o aumento do tempo de internação ${ }^{7}$. Assim, o paciente se torna vulnerável às infecções pelas modificações metabólicas relacionadas à redução do aporte calórico que leva à desnutrição, diminuição do sistema imunológico e consequente dificuldade da resposta inflamatória ${ }^{8}$.

O estudo de Moraes e col. ${ }^{9}$ analisaram evidências apresentadas no decorrer de ensaios clínicos randomizados que comprovaram a inexistência de aumento considerável do risco de complicações em procedimentos cirúrgicos e anestésicos pela abreviação do jejum. Além de não aumentar a possibilidade de danos, observou que a diminuição do tempo de jejum préoperatório está associada a benefícios no processo de recuperação do paciente.

Contudo, a relevância deste estudo decorre da necessidade de esclarecer sobre os fatores deletérios do jejum prolongado e os critérios exigidos para a redução do seu tempo, vislumbrando a redução dos efeitos deletérios da resposta orgânica ao trauma ${ }^{3,4}$. Assim, objetiva-se descrever, a partir da literatura, as recomendações atuais para a abreviação do jejum pré-operatório em cirurgias eletivas.

\section{METODOLOGIA}

Trata-se de uma revisão integrativa da literatura, que aborda a temática da abreviação do jejum pré-operatório. As etapas desta pesquisa foram analisadas com a utilização das recomendações adaptadas do Preferred Reporting Items for Systematic Reviews and MetaAnalyses (Prisma) ${ }^{10}$. A questão norteadora para seleção dos artigos foi "quais são as recomendações atuais sobre a abreviação do jejum pré-operatório?” Assim, os parâmetros definidos na estratégia de busca foram: população (paciente cirúrgico em cirurgias eletivas) 
interesse do estudo (abreviação do jejum pré-operatório), contexto (sem comparação) e desfecho (orientações atuais para a segurança do paciente).

\section{Critérios de elegibilidade}

Para a aquisição dos dados necessários na construção desta pesquisa foram incluídos artigos científicos publicados entre 2012 e 2021. Foram considerados elegíveis os textos aos quais mencionavam, obrigatoriamente, a abreviação do jejum no período pré-operatório. Excluíram-se os protocolos de pesquisa, textos indisponíveis na íntegra, editoriais, teses e dissertações.

\section{Estratégia de busca bibliográfica}

Realizou-se o levantamento bibliográfico no Portal da Biblioteca Virtual de Saúde (BVS) em outubro de 2021, o qual contempla as bases de dados Literatura Latino-americana e do Caribe em Ciências da Saúde (Lilacs), e Medical Literature Analysis and Retrieval System Online (Medline). Os descritores selecionados na busca foram os termos do Medical Subject Headings (MESH) e, também, contemplados nos Descritores em Ciências da Saúde (DeCS), a saber: "jejum”, "Cuidados pré-operatório "e "procedimentos cirúrgicos eletivos” A utilização dos descritores ocorreu de forma controlada no Portal da BVS, de modo a recuperar somente os textos disponíveis na íntegra, todos os tipos de estudos, publicados nos últimos nove anos (2012-2021), nos idiomas português, espanhol e inglês. Seguem os detalhes da pesquisa na BVS: jejum, cuidados pré-operatório, procedimentos cirúrgicos eletivos AND (db: ("MEDLINE" OR "LILACS") AND mj: ("Jejum" OR "Cuidados Pré-Operatórios" OR "Procedimentos Cirúrgicos Eletivos") AND La: ("en" OR "pt" OR "es")) AND (year_cluster: [2012 TO 2021]).

\section{Seleção e avaliação da qualidade dos estudos}

A seleção dos artigos para compor o referencial teórico exigiu leitura e análise minuciosa de todo material coletado. Todo o processo de seleção foi realizado de forma 
independente pelos autores e poucas discordâncias formam decididas por consenso. Inicialmente, identificaram-se 48 textos (primeira etapa) e a partir da leitura completa dos títulos, resumos e palavras-chave confirmou-se a exclusão de 24 artigos por não abordarem os descritores exigidos - três artigos constavam duplicados nas bases de dados Medline e Lilacs (segunda etapa). Ao final (terceira etapa), após considerar os critérios de elegibilidade temática especifica da abreviação do jejum pré-operatório em procedimento cirúrgico eletivo - restou uma amostra de dezesseis textos, sendo dois textos na base de dados Lilacs e quatorze na Medline. O resultado encontra-se descrito na Figura 1:

Figura 1 - Fluxograma da seleção dos artigos

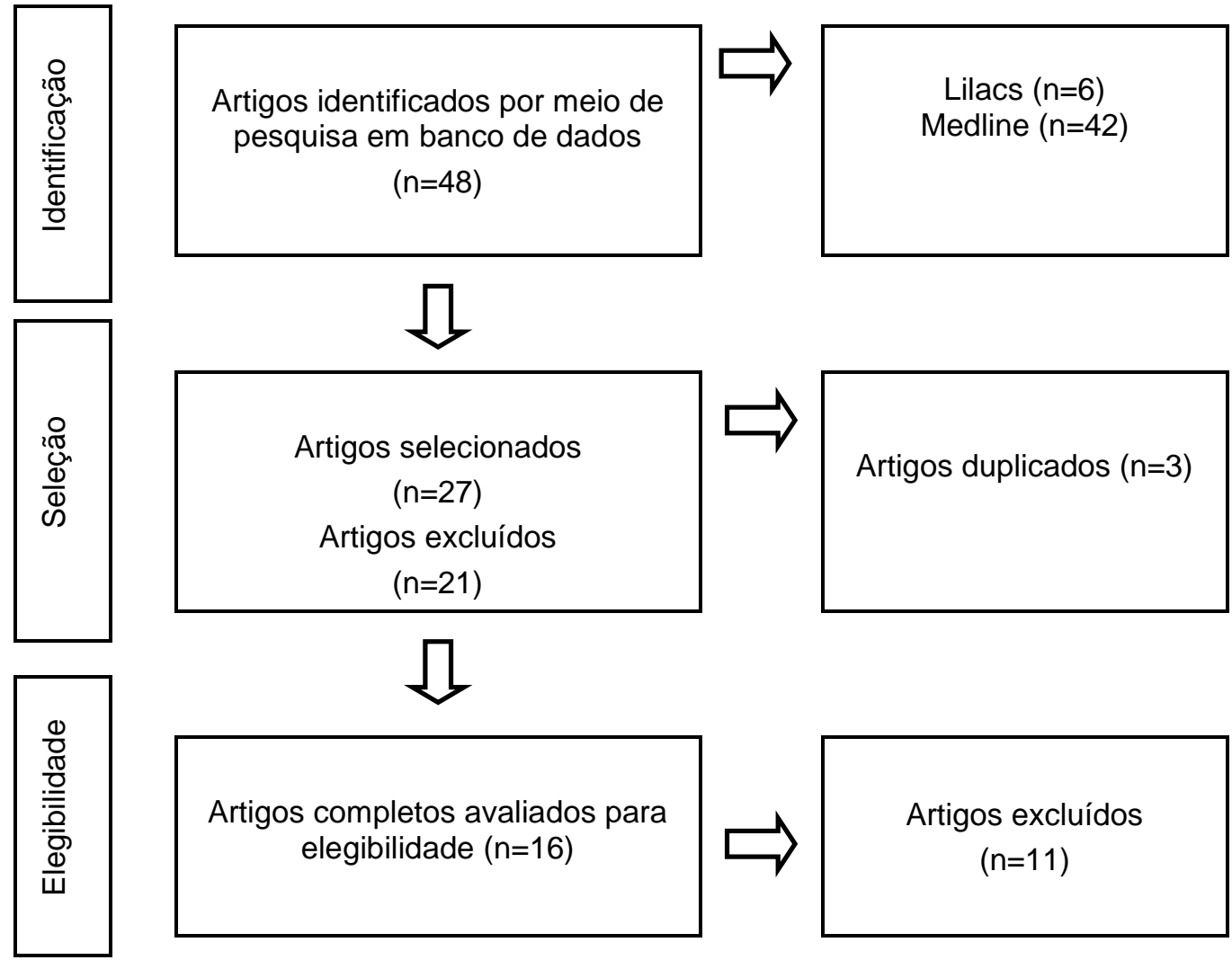

Fonte: Elaboração própria. 


\section{RESULTADOS E DISCUSSÃO}

A fim de facilitar a coleta de informações foi elaborado um quadro (Quadro 1) com os

dados sobre a abreviação do jejum pré-operatório, como as características dos textos (título do

artigo, base de dados, periódico e ano), objetivo do estudo e principais resultados e/o

contribuições dos dezesseis artigos incluídos na amostra.

Quadro 1. Artigos sobre abreviação do jejum pré-operatório

\begin{tabular}{|c|c|c|c|c|}
\hline Estudos & $\begin{array}{c}\text { Base de } \\
\text { dados }\end{array}$ & $\begin{array}{c}\text { Periódico } \\
\text { Ano }\end{array}$ & Objetivo & Resultados \\
\hline $\begin{array}{l}\text { 1. Jejum pré- } \\
\text { operatório abreviado } \\
\text { favorece } \\
\text { realimentação pós- } \\
\text { operatória com } \\
\text { menor custo de } \\
\text { internação hospitalar } \\
\text { em pacientes } \\
\text { oncológicos. } \\
\end{array}$ & Medline & $\begin{array}{l}\text { Revista do } \\
\text { Colégio } \\
\text { Brasileiro de } \\
\text { Cirurgiões. } \\
2019\end{array}$ & $\begin{array}{l}\text { Avaliar a viabilidade de } \\
\text { abreviação do jejum em } \\
\text { cirurgias colorretais } \\
\text { oncológicas, bem como o } \\
\text { impacto no desfecho } \\
\text { cirúrgico dos pacientes. }\end{array}$ & $\begin{array}{l}\text { O jejum pré-operatório abreviado } \\
\text { favorece a recuperação metabólico- } \\
\text { nutricional e diminui o tempo para } \\
\text { realimentação plena. A implantação do } \\
\text { protocolo de abreviação do jejum reduz } \\
\text { custos de internação hospitalar. }\end{array}$ \\
\hline $\begin{array}{l}\text { 2. Fasting time and } \\
\text { metabolic changes } \\
\text { in elective surgeries: } \\
\text { na integrative } \\
\text { review. }\end{array}$ & Lilacs & $\begin{array}{l}\text { Online } \\
\text { Brazilian } \\
\text { Journal de } \\
\text { Nursing. } \\
2021\end{array}$ & $\begin{array}{l}\text { Identificar na literatura a } \\
\text { ocorrência de alterações } \\
\text { metabólicas no pós- } \\
\text { operatório de cirurgias } \\
\text { eletivas e sua relação com o } \\
\text { tempo de jejum no pré- } \\
\text { operatório. }\end{array}$ & $\begin{array}{l}\text { O tempo de jejum pré-operatório } \\
\text { superior a oito horas está relacionado à } \\
\text { ocorrência de alterações metabólicas no } \\
\text { pós-operatório. }\end{array}$ \\
\hline $\begin{array}{l}\text { 3. Preoperative } \\
\text { fasting times in } \\
\text { elective surgical } \\
\text { patients at a referral } \\
\text { Hospital in } \\
\text { Botswana. }\end{array}$ & Medline & $\begin{array}{l}\text { Pan African } \\
\text { Medical } \\
\text { Journal. } \\
2016\end{array}$ & $\begin{array}{l}\text { Avaliar o jejum pré- } \\
\text { operatório, compará-lo com } \\
\text { as recomendações da ASA, } \\
\text { bem como os fatores que } \\
\text { influenciam o jejum. }\end{array}$ & $\begin{array}{l}\text { A maioria dos pacientes }(98,1 \%) \text { foi } \\
\text { orientada a jejuar a partir da meia-noite. } \\
\text { O período médio de jejum foi de } 15,9 \pm \\
2,5 \mathrm{~h} \text { (intervalo de } 12,0-25,3 \mathrm{~h} \text { ) para } \\
\text { sólidos e } 15,3 \pm 2,3 \mathrm{~h} \text { (intervalo de } \\
12,0-22,0 \mathrm{~h} \text { ) para líquidos. A duração } \\
\text { média do jejum foi significativamente } \\
\text { maior para os pacientes operados após o } \\
\text { meio-dia, em comparação com aqueles } \\
\text { operados antes do meio-dia. }\end{array}$ \\
\hline $\begin{array}{l}\text { 4. Preoperative } \\
\text { fasting for elective } \\
\text { surgery in a regional } \\
\text { hospital in Oman. }\end{array}$ & Medline & $\begin{array}{l}\text { British } \\
\text { Journal of } \\
\text { Nursing. } \\
2016\end{array}$ & $\begin{array}{l}\text { Avaliar a duração do jejum } \\
\text { antes de cirurgias eletivas } \\
\text { em pacientes adultos. }\end{array}$ & $\begin{array}{l}\text { As horas mínimas e máximas de jejum } \\
\text { em relação à alimentação foram } 7 \text { horas } \\
\text { e } 19 \text { horas, respectivamente - todos os } \\
\text { pacientes jejuaram por mais tempo do } \\
\text { que o recomendado. As horas mínimas } \\
\text { e máximas de jejum para líquidos foram } \\
4 \text { horas e } 19 \text { horas, respectivamente } \\
\text { todos os pacientes jejuaram por mais } \\
\text { tempo do que o recomendado. }\end{array}$ \\
\hline $\begin{array}{l}\text { 5. An exploration of } \\
\text { pre-operative fasting } \\
\text { practices in adult } \\
\text { patients having } \\
\text { elective surgery. }\end{array}$ & Medline & $\begin{array}{l}\text { British } \\
\text { Journal of } \\
\text { Nursing. } \\
2020\end{array}$ & $\begin{array}{l}\text { Explorar as práticas de } \\
\text { jejum pré-operatório em } \\
\text { pacientes adultos de uma } \\
\text { perspectiva internacional. }\end{array}$ & $\begin{array}{l}\text { Constatou-se que os anestesistas } \\
\text { possuem maior conhecimento sobre a } \\
\text { redução do jejum pré-operatório em } \\
\text { relação aos outros profissionais de } \\
\text { saúde, incluindor enfermeiros. } \\
\begin{array}{l}\text { Descobriu-se que o tempo real de jejum } \\
\text { é relativamente maior do que o tempo }\end{array}\end{array}$ \\
\hline
\end{tabular}




\begin{tabular}{|c|c|c|c|c|}
\hline & & & & de jejum prescrito. \\
\hline $\begin{array}{l}\text { 6. Preoperative } \\
\text { Fasting Guidelines: } \\
\text { Where Are We } \\
\text { Now? Findings } \\
\text { From Current } \\
\text { Practices in a } \\
\text { Tertiary Hospital. }^{14}\end{array}$ & Medline & $\begin{array}{l}\text { Journal of } \\
\text { PeriAnesthesi } \\
\text { a Nursing. } \\
2021\end{array}$ & $\begin{array}{l}\text { Investigar a implementação } \\
\text { das recomendações das } \\
\text { diretrizes de jejum entre } \\
\text { enfermeiros e } \\
\text { anestesiologistas, bem como } \\
\text { avaliar a duração real do } \\
\text { jejum. }\end{array}$ & $\begin{array}{l}\text { As durações reais de jejum dos } \\
\text { pacientes foram significativamente } \\
\text { maiores do que as durações de jejum } \\
\text { instruídas }(13,41 \pm 2,64 \text { vs } 9,87 \pm 2,20 \\
\text { horas, P <0,001) e líquidos claros } \\
(10,27 \pm 3,67 \text { vs } 8,98 \pm 2,90 \text { horas, } \mathrm{P} \\
<0,001) \text {. As durações instruídas pela } \\
\text { enfermeira foram significativamente } \\
\text { mais longas do que as instruídas pelo } \\
\text { anestesiologista }(9,87 \pm 2,20 \text { vs } 9,00 \pm \\
2,00 \text { horas para alimentos sólidos, P } \\
<0,001 ; 8,98 \pm 2,90 \text { vs } 6,15 \pm 3,25 \\
\text { horas para fluidos claros, } \mathrm{P}<0,001) \text {. }\end{array}$ \\
\hline $\begin{array}{l}\text { 7. Evaluating the } \\
\text { effects of } \\
\text { preoperative fasting } \\
\text { and fluid } \\
\text { limitation. }\end{array}$ & Medline & $\begin{array}{l}\text { International } \\
\text { Journal of } \\
\text { Nursing } \\
\text { Practice. } \\
2015\end{array}$ & $\begin{array}{l}\text { Avaliar os efeitos do jejum } \\
\text { pré-operatório e da limitação } \\
\text { de fluidos em pacientes } \\
\text { submetidos à } \\
\text { colecistectomia } \\
\text { laparoscópica. }\end{array}$ & $\begin{array}{l}\text { Os pacientes em jejum maior ou igual a } \\
12 \text { horas apresentaram maiores escores } \\
\text { de fome, sede, náusea e dor. O escore } \\
\text { médio de ansiedade - traço de pacientes } \\
\text { em jejum de } 12 \text { horas ou mais foi } \\
\text { estatisticamente significativamente. } \\
\text { Observou-se que não receber nada por } \\
\text { via oral após a meia-noite no pré- } \\
\text { operatório é uma intervenção } \\
\text { persistente e resulta em desconforto } \\
\text { para os pacientes. }\end{array}$ \\
\hline $\begin{array}{l}\text { 8. Ultrasound } \\
\text { assessment of } \\
\text { gastric volume in } \\
\text { children after } \\
\text { drinking } \\
\text { carbohydrate- } \\
\text { containing fluids. }\end{array}$ & Medline & $\begin{array}{l}\text { British } \\
\text { Journal of } \\
\text { Anesthesia. } \\
2016 .\end{array}$ & $\begin{array}{l}\text { Avaliar o volume gástrico } \\
\text { em crianças por meio de } \\
\text { ultrassom antes e depois de } \\
\text { beber líquidos com } \\
\text { carboidratos previamente à } \\
\text { cirurgia. }\end{array}$ & $\begin{array}{l}\text { A ingestão de líquidos com carboidratos } \\
2 \text { horas antes da cirurgia reduziu o } \\
\text { volume gástrico e não causou } \\
\text { complicações graves em pacientes } \\
\text { pediátricos. Os pais ficaram satisfeitos } \\
\text { com a bebida com carboidratos pré- } \\
\text { operatória, e as crianças tiveram } \\
\text { benefícios. }\end{array}$ \\
\hline $\begin{array}{l}\text { 9. Audit on } \\
\text { Preoperative Fasting } \\
\text { of Elective Surgical } \\
\text { Patients in an } \\
\text { African Academic } \\
\text { Medical Center. }^{17}\end{array}$ & Medline & $\begin{array}{l}\text { Wold Journal } \\
\text { Surg. } \\
2014\end{array}$ & $\begin{array}{l}\text { Avaliar a duração do jejum } \\
\text { pré-operatório para cirurgias } \\
\text { eletivas. }\end{array}$ & $\begin{array}{l}\text { A maioria dos pacientes jejuou } \\
\text { alimentos e líquidos por mais tempo do } \\
\text { que o recomendado pela maioria das } \\
\text { diretrizes internacionais. Isso pode ter } \\
\text { muitas consequências adversas, como } \\
\text { fome, sede, dor de cabeça, desidratação, } \\
\text { hipoglicemia, atraso no despertar após a } \\
\text { anestesia e má evolução do paciente } \\
\text { após a cirurgia. }\end{array}$ \\
\hline $\begin{array}{l}\text { 10. Prolonged } \\
\text { preoperative fasting } \\
\text { and prognosis in } \\
\text { critically ill } \\
\text { gastrointestinal } \\
\text { surgery patients. }\end{array}$ & Medline & $\begin{array}{l}\text { Asia Pac J } \\
\text { Clin Nutr. } \\
2020\end{array}$ & $\begin{array}{l}\text { Avaliar a influência do } \\
\text { jejum pré-operatório } \\
\text { prolongado no prognóstico } \\
\text { em cirurgias eletivas. }\end{array}$ & $\begin{array}{l}\text { Os pacientes do grupo de tempo de } \\
\text { jejum pré-operatório regular tiveram } \\
\text { menos duração do suporte de ventilação } \\
\text { mecânica após a cirurgia e as lesões } \\
\text { miocárdicas pós-operatórias (lesão } \\
\text { miocárdica } 2 \text { casos vs } 11 \text { casos, p = } \\
0,038 \text { ) e as porcentagens de reoperação } \\
\text { (reoperação } 0 \text { casos vs } 7 \text { casos, p = } \\
0,044 \text { ) foram menores em comparação } \\
\text { com o grupo de jejum pré-operatório } \\
\text { prolongado. }\end{array}$ \\
\hline $\begin{array}{l}\text { 11. Impact of } \\
\text { preoperative fasting } \\
\text { times on blood } \\
\text { glucose } \\
\text { concentration, } \\
\text { ketone bodies and } \\
\text { acid-base balance in }\end{array}$ & Medline & $\begin{array}{l}\text { European } \\
\text { Journal of } \\
\text { Anaesthesiolo } \\
\text { gy. } \\
2015\end{array}$ & $\begin{array}{l}\text { Avaliar o efeito do jejum } \\
\text { pré-operatório sobre a } \\
\text { concentração de glicose, os } \\
\text { corpos cetônicos e o } \\
\text { equilíbrio ácido-básico em } \\
\text { crianças. }\end{array}$ & $\begin{array}{l}\text { Após jejum pré-operatório prolongado, } \\
\text { crianças menores de } 36 \text { meses podem } \\
\text { apresentar cetoacidose e baixas } \\
\text { concentrações de glicose no sangue. Os } \\
\text { tempos reais de jejum devem ser } \\
\text { otimizados de acordo com as diretrizes } \\
\text { existentes. Em bebês pequenos, os }\end{array}$ \\
\hline
\end{tabular}




\begin{tabular}{|c|c|c|c|c|}
\hline $\begin{array}{l}\text { children younger } \\
\text { than } 36 \text { months: A } \\
\text { prospective } \\
\text { observational } \\
\text { study. }{ }^{18}\end{array}$ & & & & $\begin{array}{l}\text { desvios das diretrizes de jejum devem } \\
\text { ser os mais curtos possíveis - não mais } \\
\text { do que } 2 \text { horas. }\end{array}$ \\
\hline $\begin{array}{l}\text { 12. Fasting Time in } \\
\text { the Preoperative of } \\
\text { Elective Surgeries at } \\
\text { a Referral Hospital } \\
\text { in Trauma in the } \\
\text { Municipality of } \\
\text { Fortaleza - CE. }^{20}\end{array}$ & Lilacs & $\begin{array}{l}\text { Revista } \\
\text { Brasileira de } \\
\text { Ciências da } \\
\text { Saúde. } \\
2019\end{array}$ & $\begin{array}{l}\text { Analisar o tempo de jejum } \\
\text { no pré-operatório de } \\
\text { cirurgias eletivas em um } \\
\text { hospital de referência do } \\
\text { Município de Fortaleza. }\end{array}$ & $\begin{array}{l}\text { Os pacientes foram submetidos a um } \\
\text { tempo médio de jejum pré-operatório } \\
\text { superior às recomendações da American } \\
\text { Society of Anesthesiologists. Esse } \\
\text { período foi igualmente extenso para a } \\
\text { ingestão prévia de sólidos e líquidos. }\end{array}$ \\
\hline \begin{tabular}{l|} 
13. Preoperative \\
Fasting Time and Its \\
Association with \\
Hypoglycemia \\
during Anesthesia in \\
Pediatric Patients \\
Undergoing Elective \\
Procedures at Tikur \\
Anbessa Specialized \\
Hospital, Addis \\
Ababa, Ethiopia.
\end{tabular} & Medline & \begin{tabular}{l|} 
BioMed \\
Research \\
International. \\
2021.
\end{tabular} & $\begin{array}{l}\text { Avaliar o tempo de jejum } \\
\text { pré-operatório e sua } \\
\text { associação com } \\
\text { hipoglicemia durante a } \\
\text { anestesia em pacientes } \\
\text { pediátricos submetidos a } \\
\text { procedimentos eletivos no } \\
\text { Hospital Especializado } \\
\text { Tikur Anbessa, Addis } \\
\text { Abeba, Etiópia. }\end{array}$ & 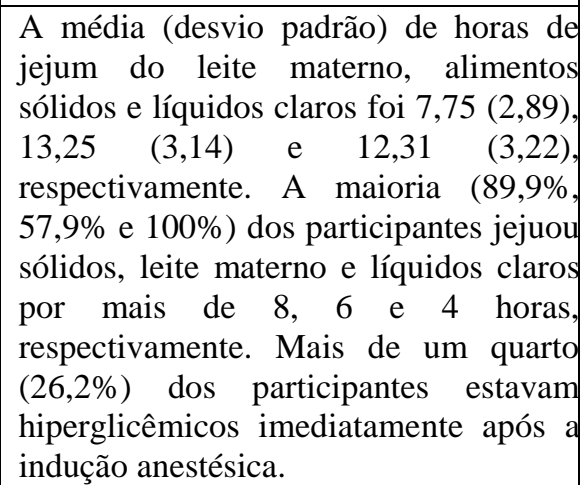 \\
\hline $\begin{array}{l}\text { 14. Mudando } \\
\text { paradigmas em } \\
\text { jejum pré- } \\
\text { operatório: } \\
\text { resultados de um } \\
\text { mutirão em cirurgia } \\
\text { pediátrica. }^{22}\end{array}$ & Medl & \begin{tabular}{l|} 
ABCD \\
Arquivos \\
Brasileiros de \\
Cirurgia \\
Digestiva. \\
2017
\end{tabular} & $\begin{array}{l}\text { Avaliar a segurança de um } \\
\text { protocolo de abreviação do } \\
\text { jejum pré-operatório, com o } \\
\text { uso de bebida contendo } \\
\text { carboidratos, e } \\
\text { realimentação precoce. }\end{array}$ & $\begin{array}{l}\text { O tempo mediano de jejum pré- } \\
\text { operatório foi de } 145 \text { min e o tempo } \\
\text { mediano para realimentação foi de } 135 \\
\text { min. Não houve eventos adversos } \\
\text { durante a anestesia ou operação. }\end{array}$ \\
\hline $\begin{array}{l}\text { 15. Preoperative } \\
\text { fasting - "nihil per } \\
\text { os" a difficult myth } \\
\text { to break down: a } \\
\text { randomized } \\
\text { controlled study. }\end{array}$ & Medline & $\begin{array}{l}\text { G Chir. } \\
2020\end{array}$ & $\begin{array}{l}\text { Identificar a extensão da } \\
\text { lacuna que existe entre o } \\
\text { tempo de jejum pré- } \\
\text { operatório necessário e } \\
\text { aquele realmente encontrado } \\
\text { em salas de cirurgia. }\end{array}$ & $\begin{array}{l}\text { O uso de bebidas enriquecidas com } \\
\text { carboidratos até } 2 \text { horas após a indução } \\
\text { anestésica parece ser um procedimento } \\
\text { seguro. O uso dessas soluções reduz a } \\
\text { resposta catabólica à cirurgia e } \\
\text { contribui para a manutenção de um } \\
\text { estado de bem-estar pré-operatório. }\end{array}$ \\
\hline $\begin{array}{l}\text { 16. Reducing } \\
\text { preoperative fasting } \\
\text { in elective adult } \\
\text { surgical patients: a } \\
\text { case-control study. }\end{array}$ & Medline & $\begin{array}{l}\text { Ir J Med Sci. } \\
2012 .\end{array}$ & $\begin{array}{l}\text { Avaliar o impacto de um } \\
\text { protocolo baseado em } \\
\text { evidências para redução do } \\
\text { jejum pré-operatório nos } \\
\text { tempos de jejum, segurança } \\
\text { e conforto do paciente. }\end{array}$ & $\begin{array}{l}\text { Reduções significativas nos tempos de } \\
\text { jejum para líquidos e sólidos foram } \\
\text { alcançadas após a implementação do } \\
\text { protocolo. Foram menos relatadas sede } \\
\text { pré-operatória, cefaleia e náuseas por } \\
\text { aqueles que fizeram um jejum mais } \\
\text { curto. A broncoaspiração } \\
\text { intraoperatória não ocorreu em nenhum } \\
\text { dos grupos. }\end{array}$ \\
\hline
\end{tabular}

Após, realizou-se a análise crítica e categorização temática para a composição do corpus da discussão, a saber: prejuízos do jejum pré-operatório prolongado em cirurgias eletivas, segurança da abreviação do jejum e seus benefícios, e recomendações para a abreviação do jejum pré-operatório. 


\subsection{Prejuízos do jejum pré-operatório prolongado em cirurgias eletivas}

Previamente à realização de algum procedimento cirúrgico-anestésico é necessário o período de jejum para evitar a aspiração pulmonar de conteúdo estomacal. Os protocolos de recomendações do jejum pré-operatório para pacientes saudáveis recomendam de seis horas para alimentos sólidos (leves) e duas horas para líquidos. No entanto, muitos hospitais praticam o "nada-pela-boca" após a meia-noite, o que afeta a recuperação do paciente e aumenta o tempo de permanência no hospital ${ }^{11}$. Nesse sentido, estudo ${ }^{12}$ verificou que o jejum excessivo pode causar desconforto e morbidade em pacientes cirúrgicos.

Destaca-se que em muitas ocasiões os pacientes não recebem as devidas orientações sobre o jejum pré-operatório, e os pacientes acreditam que quanto maior o jejum menor é o risco de complicações. Estudo ${ }^{13}$ evidencia que os pacientes com cirurgias marcadas para o período matutino e vespertino recebiam o ultimo alimento no mesmo horário, ou seja, os pacientes agendados para a tarde ficaram um período bem extenso de jejum - estes pacientes reclamaram de desconforto moderado e intenso (fome e sede) no pré e pós-operatório. Em outro estudo $^{14}$ as práticas do jejum pré-operatório orientadas por enfermeiros na prática clínica eram mais longas do que estes profissionais entendiam como sendo o tempo ideal, sendo que os anestesiologistas também prescreveram jejuns mais longos do que a duração mínima de jejum recomendada.

Os períodos prolongados de jejum são desnecessários e podem causar complicações ao paciente, como desidratação; desequilíbrio eletrolítico; angústia e confusão mental; hipoglicemia; náuseas e vômitos pós-operatórios; e aumento da resistência à insulina. Além disso, sintomas pré-operatórios como cefaleia, náuseas, sede e fome estão seriamente associados à duração do jejum prolongado ${ }^{11}$. Pesquisa ${ }^{15}$ que avaliou os efeitos do jejum préoperatório e a limitação de fluidos em pacientes submetidos à colecistectomia laparoscópica eletiva constatou que os pacientes que tiveram um jejum de doze horas ou mais apresentaram 
desconfortos (fome, dor, náusea, sede e sonolência) e que alguma ansiedade. Além disso, o jejum prolongado está associado à desidratação perioperatória, irritabilidade e hipotensão ${ }^{16}$; e pode causar atraso no despertar após a anestesia e má evolução do paciente após a cirurgia ${ }^{17}$.

Sobre a questão, Zhou e col. ${ }^{18}$ estudaram o jejum pré-operatório prolongado em pacientes graves de cirurgia gastrointestinal, onde os dividiram em dois grupos: jejum préoperatório regular - duas e seis horas - e jejum pré-operatório prolongado - quatro e oito horas. Como resultados, no grupo de jejum prolongado teve maior duração do suporte de ventilação mecânica após a cirurgia e mais pacientes apresentaram lesões miocárdicas pósoperatórias, ou seja, o jejum prolongado levou a resultados desfavoráveis.

Ademais, ao considerar que o extenso período de jejum pré-operatório e a ausência de diferenciação entre a orientação de jejum líquido e sólido são práticas prejudiciais à saúde, a American Society of Anesthesiologists (ASA) sugere o tempo mínimo de jejum ${ }^{19}$, que é descrita por Abebe e col $^{11}$ e Moraes e col. ${ }^{9}$ no Quadro 2.

Quadro 2. Recomendações de jejum pré-operatório

\begin{tabular}{|l|l|}
\hline Tipo de alimento & Tempo mínimo de jejum \\
\hline Líquidos sem resíduos & 2 horas \\
\hline Leite materno & 4 horas \\
\hline Fórmula infantil & 6 horas \\
\hline Leite não materno & 6 horas \\
\hline Dieta leve & 6 horas \\
\hline Carnes e frituras & 8 horas \\
\hline
\end{tabular}

Fonte: Abebe e col ${ }^{11}$, Moraes e col. ${ }^{9}$

Pesquisa $^{20}$ realizada em um hospital do Ceará-Brasil ${ }^{20}$ verificou o tempo de jejum préoperatório de cirurgias eletivas e constatou que os pacientes foram submetidos ao tempo médio de jejum superior ao sugerido pelo Protocolo ASA - prejudicando a recuperação no pós-operatório devido às alterações metabólicas. Nesse sentido, estudo de Moraes e col. ${ }^{9}$ descrevem algumas alterações que são descritas no Quadro 3.

Quadro 3. Alterações metabólicas com o jejum pré-operatório com 8hs ou mais 


\begin{tabular}{|l|l|}
\hline Marcador biológico & Principais alterações \\
\hline Glicose & $\begin{array}{l}\text { Elevação progressiva do nível sérico de glicose do intraoperatório até as } \\
\text { primeiras três horas pós craniotomia eletiva. Elevação progressiva do nível de } \\
\text { glicose a partir do pós-operatório imediato com queda 24 horas após } \\
\text { colecistectomia laparoscópica. }\end{array}$ \\
\hline \multirow{3}{*}{ Insulina plasmática } & $\begin{array}{l}\text { Elevação do nível de insulina no intraoperatório com queda progressiva a partir } \\
\text { de 2 horas pós-procedimento, retornando aos níveis basais três horas após } \\
\text { craniotomia. Elevação progressiva do nível de insulina do pós-operatório } \\
\text { imediato até 24 horas após colecistectomia laparoscópica. }\end{array}$ \\
\hline Cortisol & $\begin{array}{l}\text { Elevação dos níveis plasmáticos de cortisol duas horas após a incisão cutânea } \\
\text { em discectomia lombar. Aumento plasmático de cortisol imediatamente após } \\
\text { colecistectomia laparoscópica com queda expressiva 24 horas após a finalização } \\
\text { do procedimento. }\end{array}$ \\
\hline IGF-I, IGFBP-3-PA & $\begin{array}{l}\text { Diminuição significativa de IGF-I e na proteólise do IGFBP-3, com o } \\
\text { consequente aumento da resistência insulínica pós-operatória em submetidos à } \\
\text { cirurgia abdominal. }\end{array}$ \\
\hline Interleucina 6 (IL-6) & Aumento dos níveis de IL-6 no pós-operatório mediato de discectomia lombar. \\
\hline
\end{tabular}

Fonte: Moraes e col. ${ }^{9}$

Ao avaliar o tempo de jejum pré-operatório e sua associação com hipoglicemia durante anestesia em pacientes pediátricos submetidos aos procedimentos cirúrgicos, estudo ${ }^{21}$ constatou que a maioria das crianças foi submetida ao jejum de sólidos, leite materno e líquidos claros por mais de oito, seis e quatro horas respectivamente; e, por isto, mais de um quarto $(26,2 \%)$ delas estavam hipoglicêmicas imediatamente após a indução anestésica.

Contudo, o jejum pré-operatório prolongado pode ser bem perigoso para as crianças, pois o impacto dos tempos de jejum na concentração de glicose no sangue, corpos cetônicos e equilíbrio ácido-básico. Os tempos reais de jejum devem ser otimizados de acordo com as diretrizes existentes, e nos casos de bebês os desvios das diretrizes de jejum devem ser os mais curtos possíveis e não maior do que duas horas ${ }^{19}$.

\subsection{Segurança da abreviação do jejum e seus benefícios}

No Brasil existe o Projeto Aceleração da Recuperação Total Pós-operatória (Acerto) ${ }^{9}$, que explica que o esvaziamento gástrico para líquidos não calóricos ocorre de maneira rápida (aproximadamente dez minutos metade da quantidade ingerida passa para o duodeno), e quando o líquido é enriquecido com glicose o esvaziamento é inicialmente mais lento, porém 
decorridos 90 minutos não existe diferença. Para os sólidos, o esvaziamento inicia-se em uma hora, e com duas horas aproximadamente $50 \%$ ultrapassa o estômago para o duodeno, independe da quantidade ingerida (dependente da densidade calórica). Assim, este Protocolo considera seguro a ingestão de líquidos claros até duas horas antes do procedimento anestésico; já para os preparados que incluem leite ou outra fonte de gordura é preciso de seis horas; bem como para os alimentos sólidos.

Através do Protocolo Acerto vários estudos foram realizados e observou-se a possibilidade de os pacientes tomarem soluções de carboidratos, acrescidos ou não de proteínas, no período de até três horas antes da cirurgia - os resultados demostraram que esta prática é segura e melhora os desfechos da cirurgia 9 .

Estudo ${ }^{16}$ avaliou o volume gástrico em crianças através de ultrassonografia, sendo que na avaliação inicial foi realizada após jejum de oito e duas horas antes da cirurgia (os pacientes receberam bebidas com carboidratos). Antes da indução da anestesia geral, o volume gástrico foi reavaliado, com a constatação de que a ingestão de líquidos com carboidratos duas horas antes da cirurgia reduziu o volume gástrico e não causou complicações graves em pacientes pediátricos (a bebida aliviou a sede e a irritabilidade).

Assim, os benefícios da abreviação do jejum não são válidos apenas para os adultos, sendo que em estudo de Carvalho e col. ${ }^{22}$ as crianças receberam suplemento nutricional com $150 \mathrm{ml}$ de maltodextrina $(12,5 \%)$ aproximadamente duas horas antes do procedimento. Constatou-se que a abreviação de jejum em algumas cirurgias eletivas de crianças - fimose, hérnia inguinal, hérnia umbilical e criptorquidia - é seguro e não está associado ao maior risco de broncoaspiração pulmonar. Estudo ${ }^{23}$ semelhante realizado com indivíduos adultos afirmou ser seguro a administração de bebidas enriquecidas com carboidratos até duas horas antes da indução anestésica. Além disso, o uso dessas soluções reduz a resposta catabólica à cirurgia e contribui para a manutenção de um estado de bem-estar pré-operatório. 
Em outra pesquisa ${ }^{5}$ que tratou da viabilidade de abreviação do jejum em cirurgias colorretais oncológicas, observou-se que o jejum abreviado ajudou na recuperação fisiológica e no retorno precoce à dieta plena, ou seja, o jejum abreviado revelou-se como fator principal com significância estatística para o retorno à dieta plena. Ademais, Power e col. ${ }^{24}$ constataram que abreviar o jejum de líquidos e sólidos para duas e seis horas antes da anestesia em cirurgias eletivas reduziu a sede pré-operatória, cefaleia e náuseas.

\subsection{Recomendações para a abreviação do jejum pré-operatório}

Muitas evidências sugerem que um estômago normal e saudável esvazia os líquidos claros rapidamente e, por isto, torna-se seguro beber líquidos claros até duas horas antes da indução da anestesia e ingerir alimentos sólidos até seis horas antes da cirurgia. Portanto, a ordem "nada pela boca" começando à meia-noite para cirurgia eletiva pode ser ajustada para nenhuma ingestão de alimentos sólidos e produtos lácteos seis horas antes da cirurgia, e jejum de líquido claro duas horas antes da cirurgia. ${ }^{14,15,17}$

Observa-se que diferentes diretrizes recomendam o período mínimo de jejum de duas horas para líquidos claros e seis horas para sólidos ${ }^{11,12,13,17,20}$. No entanto, Assen e col. ${ }^{21}$ descreveram que a Sociedade Europeia de Anestesiologistas implantou um protocolo de jejum pré-operatório mais liberal para líquidos claros (uma hora antes da anestesia).

Dennhardt e col. ${ }^{19}$ afirmaram que alguns estudos apontam tempos de jejum mais curtos (por exemplo, quatro horas para uma refeição leve ou fórmula infantil, três horas para o leite materno e duas horas para fluidos claros) sem relatar uma maior incidência de aspiração pulmonar. Os mesmos autores afirmam que a abreviação do jejum com fluidos claros uma hora antes do procedimento cirúrgico não altera o pH gástrico ou o volume residual em comparação com o jejum de duas horas.

Através da implantação do protocolo Acerto ${ }^{9}$ alguns estudos tiveram ótimos resultados quando os pacientes usaram soluções de carboidratos três horas antes do procedimento 
cirúrgico. Assim, é seguro abreviar o jejum com uma dose de Maltodextrina 12,5\%, $150 \mathrm{ml}$ duas horas antes dos procedimentos eletivos de pequeno ou médio porte. ${ }^{5,16,22,23,24}$

\section{CONCLUSÃO}

A alimentação tanto no período pré quanto no pós-operatório possui um papel importante, visto que ela ajuda a reduzir a resposta orgânica ao estresse e incômodos que interferem de maneira significativa na evolução dos pacientes.

Atualmente as sociedades de anestesiologia de todo mundo recomendam regras mais liberais sobre o jejum pré-operatório, ou seja, os protocolos têm permitido o uso de líquidos claros até duas horas antes da operação.

Após a análise dos estudos verificou que o jejum pré-operatório prolongado em cirurgias eletivas pode acarretar alguns prejuízos para o paciente como desconforto préoperatório, morbidade e alterações metabólicas, mas por outro lado a abreviação do jejum préoperatório traz benefícios como a redução do desconforto pré-operatório e redução do tempo de internação. Assim, a literatura afirma ser possível a abreviação segura do jejum préoperatório em até uma hora com o uso de líquidos claros, ou em até duas horas com a utilização de soluções de carboidratos.

\section{REFERENCIAS}

1. Carvalho PA, Göttems LBD, Pires MRGM, Oliveira MLC. Safety culture in the operating room of a public hospital in the perception of healthcare professionals1. Rev. Lat-Am [periódico na Internet]. 2015 Nov-Dec [acessado 2021 out 20]; 23(6): 1041-1048. Disponível em: https://doi.org/10.1590/0104-1169.0669.2647.

2. Martins FZ, Dall'Agnol CM. Centro cirúrgico: desafios e estratégias do enfermeiro nas atividades gerenciais. Rev Gaúcha Enferm [periódico na Internet]. 2017 Ago [Acessado 
2021

Out

20];

37(04):146-54.

Disponível

em:

https://doi.org/10.1590/19831447.2016.04.56945.

3. Campos SBG, Barros-Neto JA, Guedes GS, Moura FA. Pre-operative fasting: why abbreviate?. ABCD Arq Bras Cir Dig [periódico na Internet]. 2018 Mar [acessado 2021 Out 20]; 31(02):249-55. Disponível em: https://doi.org/10.1590/0102672020180001e1377.

4. Marquini GV, Pinheiro FES, Vieira AUC, Pinto RMC, Uyeda MGBK, Girão MJBB, et al. Efeitos da abreviação de jejum pré-operatório na incidência de náuseas e vômitos em pacientes cirúrgico-ginecológicas. Rev Bras Ginecol Obste [periódico na Internet]. 2020 Ago [acessado 2021 Out 20]; 42(08):468-475. Disponível em: https://doi.org/10.1055/s$\underline{0040-1712994 .}$

5. Reis PGA, Polakowski C, Lopes M, Bussyguin DS, Ferreira RP, Preti, VB, Tomasich, FDS. Jejum pré-operatório abreviado favorece realimentação pós-operatória com menor custo de internação hospitalar em pacientes oncológicos. Rev Col Bras Cir [periódico na Internet]. 2019 Jan [acessado 2021 Out 20]; 46(3):174-84. Disponível em: https://doi.org/10.1590/0100-6991e-20192175.

6. Silva AH, Miguez BB, Oliveira AP, Ferreira JM. A importância da redução do tempo de jejum pré-operatório: uma revisão literária. Cad bras med [periódico na Internet]. 2019 Mar [acessado 2021 Out 26]; 2(2):174-182. Disponível em: http://www.revista.unifeso.edu.br/index.php/cadernosdemedicinaunifeso/article/view/138 $\underline{9}$.

7. Carvalho CALB, Carvalho ADOG, Nogueira PLB, Mendes KBV, Nascimento DBD, Nascimento JEA. Benefícios metabólicos e inflamatórios da abreviação do jejum préoperatório em cirurgia pediátrica. Rev Col Bras Cir [periódico na Internet]. 2020 Jan 
[acessado 2021 Out 26]; 47:633-48. Disponível em: https://doi.org/10.1590/0100-6991e$\underline{20202353}$.

8. Alves DR, Ribeiras R. O jejum influencia a responsividade à pré-carga em voluntários ASA I e II? Rev Bras Anestesiol [periódico na Internet]. 2017 Mar-Abr [acessado 2021 Out 26]; 7(2):172-179. Disponível em: https://doi.org/10.1016/j.bjane.2015.11.002

9. Moraes HSC, Fassarella CS, Camerini FG, Meneses RO, Bosco PS. Fasting time and metabolic changes in elective surgeries: na integrative review. Onl Braz Jour Nur [periódico na Internet]. 2021 Jan [acessado 2021 Out 26]; 20(01):NA. Disponível em: https://docs.bvsalud.org/biblioref/2021/06/1224134/6480-pt.pdf.

10. Galvão TF, Pansani TSA, Harrard D. Principais itens para relatar revisões sistemáticas e meta-análises: a recomendação prisma. Epidemiol Serv Saúde [periódico na Internet]. 2015 Jun [acessado 2021 Out 16]; 24(2): 335-342. Disponível em: https://doi.org/10.5123/s1679-49742015000200017

11. Abebe WA, Rukewe A, Bekele NA, Stoffel M, Dichabeng MN, Shifa JZ. Preoperative fasting times in elective surgical patients at a referral Hospital in Botswana. Pan Afr Med J [periódico na Internet]. 2016 Mar [acessado 2021 Nov 18]; 23(102):104-18 Disponível em: doi: 10.11604/pamj.2016.23.102.8863.

12. Maqbali M. A.Preoperative fasting for elective surgery in a regional hospital in Oman. Br J Nur [periódico na Internet]. 2016 Jul [acessado 2021 Nov 18]; 25(14):798-802. Disponível em: https://doi.org/10.12968/bjon.2016.25.14.798.

13. Denkyi L. An exploration of pre-operative fasting practices in adult patients having elective surgery. British Journal of Nursing [periódico na Internet]. 2020 Abr [acessado 2021 24]; 29v(7):436-441. Disponível em: https://doi.org/10.12968/bjon.2020.29.7.436. 
14. Qianquian Z, Li Y, Deng Y, Zhao S, Bao K, Lai L. Preoperative Fasting Guidelines: Where Are We Now? Findings From Current Practices in a Tertiary Hospital. J PeriAnesth Nur [periódico na Internet]. 2021 Aug [acesso 2021 Nov 24]; 36(4): 388-392. Disponível em: https://doi.org/10.1016/j.jopan.2020.09.002.

15. Tuson BRN, Yava A, Açikel C. Evaluating the effects of preoperative fasting and fluid limitation. Int J Nur Pract [periódico na Internet]. 2015 Abril [acessado Nov 18]; 21(2): 156-65. Disponível em: https://doi.org/10.1111/ijn.12239.

16. Song IK, Kim HJ, Lee JH, Kim EH, Kim JT, Kim HS. Ultrasound assessment of gastric volume in children after drinking carbohydrate-containing fluids. Br J Anesth [periódico na Internet]. 2016 Apr [acessado 2021 No 18]; 116(4):513-7. Disponível em: DOI: https://doi.org/10.1093/bja/aew031.

17. Gebremedhn EG. Nagaratnam VB. Audit on Preoperative Fasting of Elective Surgical Patients in an African Academic Medical Center. World J Surg [periódico na Internet]. 2014 Sep [acessado 2021 Nov 18]; 38(9):2200-4. Disponível em: https://doi.org/10.1007/s00268-014-2582-3.

18. Zhou G, Zhu F, Na Y, Qin L, Jie LV, Zhao X, Shen J. Prolonged preoperative fasting and prognosis in critically ill gastrointestinal surgery patients Asia Pac. J Clin Nutr [periódico na Internet] 2020 Jan [acessado 2021 Nov 24]; 29(1): 41-47. Disponível em:

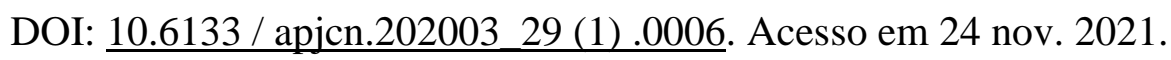

19. Dennhardt N, Beck C, Huber D, Nikel K, Sander B, Witt LH, at al. Impact of preoperative fasting times on blood glucose concentration, ketone bodies and acid-base balance in children younger than 36 months: A prospective observational study. Eur J Anaesthesiol [periódico na Internet]. 2015 Dec [acessado 2021 Nov 24]; 32(12):857-61. Disponível em: https://journals.lww.com/ejanaesthesiology/Fulltext/2015/12000/Impact_of_preoperative fasting times_on_blood.7.aspx. 
20. Diógenes D, Costa CS, Rivanor RLC. Fasting time in the preoperative of elective surgeries at a referral hospital in trauma in the municipality of Fortaleza - CE. R Bras C Saúde [periódico na Internet]. 2019 Jun [acessado 2021 Nov 17]; 23(2):191-196. Disponível em: https://doi.org/10.22478/ufpb.2317-6032.2019v23n2.32118.

21. Assen HE, Hassen AM, Abate A, Liyew B. Preoperative fasting time and its association with hypoglycemia during anesthesia in pediatric patients undergoing elective procedures at Tikur Anbessa Specialized Hospital, Addis Ababa, Ethiopia. BioMed Res Int [periódico na Internet]. 2021 Jul [acessado Nov 24]; 2021(21):203-12. Disponível em: https://www.hindawi.com/journals/bmri/2021/9166603/.

22. Carvalho CAB, Carvalho AA, Nogueira PLB, Nascimento JEA. Mudando paradigmas em jejum pré-operatório: resultados de um mutirão em cirurgia pediátrica. ABCD arq bras cir dig [periódico na Internet]. 2017 Jan-Mar [acessado 2021 Nov 18]; 30(1): 121-32. Disponível em: https://doi.org/10.1590/0102-6720201700010003.

23. Panebianco A, Laforgia R, Volpi A, Punzo C, Vacca G, Minafra M, et al. Preoperative fasting - "nihil per os" a difficult myth to break down: a randomized controlled study. G Chir [periódico na Internet]. 2020 Jan-Fev [acessado 2021 Nov 24]; 41(1):84-93. Disponível em: https://pubmed.ncbi.nlm.nih.gov/32038017/. Acesso em: 24 nov. 2021.

24. Power S, Kavanagh DO, Mcconnell G, Gronin K, Corish C, Leonard M, et al. Reducing preoperative fasting in elective adult surgical patients: a case-control study. Ir J Med Sci [periódico na Internet]. 2012 Mar [acessado 2021 Nov 24]; 181(1): 99-104. Disponívem em: https://link.springer.com/article/10.1007\%2Fs11845-011-0765-6. 